\section{How does the NPM1 mutant induce leukemia?}

\author{
Paolo Sportoletti \\ Hematology and Clinical Immunology \\ Section, Department of Clinical \\ and Experimental Medicine, \\ University of Perugia, Perugia, Italy
}

\section{Abstract}

NPM1 is the most frequently mutated gene in AML and the role of the NPM1 mutant in acute myeloid leukemia along with its leukemogenic potential are still under investigation.

NPM1 genetic alterations can contribute to leukemogenesis through the direct oncogenic effect of the mutant protein and the concomitant loss of one functional allele. Npm1 loss determines tumor development in the mouse while in human NPM1 maps in a chromosomal region frequently loss in myelodysplastic syndrome (MDS). The NPM1 mutant cytoplasmic delocalization in leukemic blasts alters multiple cellular pathways through either loss or gain of function effects on different protein partners.

Here we discuss the most relevant studies on the role of the NPM1 molecule in hematological malignancies and both in vitro and in vivo studies that are trying to elucidate the way by which the NPM1 mutation induces leukemia.

\section{Introduction}

NPM1 is a multifunctional phosphoprotein that encodes for a number of functional domains through which the molecule is able to bind many partners in distinct cellular compartments. ${ }^{1}$ NPM displays nucleolar localization and constantly shuttles between the nucleus and the cytoplasm. The shuttling activity of NPM1 along with its capacity to interact with many partners involves the protein in multiple cellular functions.

These functions include ribosome biogenesis and the transport of pre-ribosomal particles, maintenance of genomic stability through the control of cellular ploidy and centrosome duplication and the control of cellular proliferation. NPM1 is also a survival factor and participates in DNA-repair processes. In addition, NPM1 is involved in regulating the activity and stability of crucial tumor suppressors such as ARF and p53. The NPM1 polypeptide chain has a modular structure containing distinct sequence motifs including a nuclear-export signal (NES) and a nucleolar localization signal (NuLS) that resides in the C-terminal region of the protein. In acute myeloid leukemia (AML) patients the NuLS is the target of the C-terminal mutation that determines its substitution into an extra NES, thus resulting in an aberrantly localized protein that resides in the cytoplasm of leukemic blasts. ${ }^{2}$ The shuttling activity of NPM1 and its proper sub cellular localization are thought to be crucial for normal cellular homeostasis and the identification of the NPM1 mutant counterpart in AML patients has emphasized this hypothesis. Thus, genetic alteration of NPM1 can contribute to oncogenesis by directly affecting NPM1 functions.

NPM1 has been directly implicated in human cancer.1 In fact the NPM1 protein is overexpressed in solid tumours of different origin like gastric, colon, ovarian and prostate carcinomas. NPM1 is one of the most frequent targets of genetic alterations in haematopoietic tumours. It is found translocated with distinct partner genes in several diseases such as acute promyelocytic leukemia (APL), anaplastic large cell lymphoma (ALCL), acute myeloid leukemia (AML) and myelodysplasia. Moreover, the region of chromosome 5 that NPM1 maps to, is a genetic target of deletions in both de novo and therapy related MDS, a preleukemic condition with ineffective production of myeloid blood cells. More recently NPM1 has been found mutated and aberrantly localized in the cytoplasm of myeloid blasts in a high proportion of AML patients with normal karyotype. ${ }^{2}$ Although the NPM1 gene was strongly implicated in cancer pathogenesis, how the cytoplasmic localized NPM1 mutant protein (NPMc+) promotes leukemia is still under investigation.

\section{NPM1 gene alterations}

\section{contributing to leukemogenesis}

The alteration of NPM1 in human cancer can lead to tumor development as a result of two major mechanisms involving both the presence of a mutated product and the reduction of the wild type NPM1 dosage and level of expression. Indeed, NPM1 gene alterations lead to the generation of mutated products associated with hematopoietic malignancies (in the case of AML the NPMc+ mutant), and to the concomitant loss of one functional allele of the gene leading to NPM1 heterozygosity.

The reduction of the NPM dosage to heterozygosity has been shown to lead to altered NPM1 functions and tumour susceptibility ${ }^{3}$ indicating that normal levels of expression are required for the cell to prevent tumor development. Moreover mutated products retain the ability to heterodimerize with the residual wild-type protein, and they potentially interfere with its functions. In particular the dominant-negative effect of the mutated NPM determines the delocalization of the remaining
Correspondence: Paolo Sportoletti, Hematology and Clinical Immunology Section, Department of Clinical and Experimental Medicine, University of Perugia, Perugia, Italy.

E-mail: sportolp@gmail.com

Key words: NPM1, leukemia.

Received for publication: 4 May 2011. Accepted for publication: 4 June 2011.

This work is licensed under a Creative Commons Attribution NonCommercial 3.0 License (CC BYNC 3.0).

(c) Copyright P. Sportoletti, 2011

Licensee PAGEPress, Italy

Pediatric Reports 2011; 3(s2):e6

doi:10.4081/pr.2011.s2.e6

wild-type NPM protein and exacerbates the NPM functional loss. On the other end there is the capability for the NPMc+ mutant itself to function as activated oncogene. Since the NPM1 mutation always results in aberrant cytoplasmic dislocation of the mutant protein, this event appears critical for leukemogenesis. The increased NPM1 export into the cytoplasm can affect multiple cellular pathways and drive leukemia by either a loss-of-function 4,5 or a gain-of-function. ${ }^{6}$ In fact, the NPMc+ nucleolar interactors can be delocalized by the mutant into leukemic cells cytoplasm and their activity can be significantly impaired. On the other end the NPMc+ mutant may acquire the capability to interact with new protein partners in the cytoplasm. All these activities can lead to leukemia and there are a number of in vitro and in vivo data supporting this hypothesis that will be analyzed in the next paragraphs.

\section{The role of Npm functional loss in tumor development}

Data on the role of Npm functional loss in tumor development arise from mouse work that has been done in the Pandolfi's lab in the last five years. Npm complete knockout resulted in embryonic lethality due to developmental abnormalities of the brain and of the hematopoietic system. ${ }^{3}$ In this setting Npm inactivation was able to determine cancer susceptibility in vitro. The other main observation of this study implicated Npm in the pathogenesis of human MDS. Npm heterozygous mice showed some of the features found in the human syndrome. Peripheral blood showed significantly increased MCV and RDW values while the bone marrow presented a high proportion of dysplastic erythroid precursors and megakaryocyte dyspoiesis. This MDS status evolved into overt leukemia as in humans, demonstrating that Npm act as tumor suppressor gene in vivo.7 In fact, Npm1 heterozy- 
gous mice displayed higher susceptibility to develop tumor than their wild type counterparts (29\% vs $6,3 \%)$. Pathological analysis revealed that $75 \%$ of the diseased mice were affected by hematological malignancies, including myeloid leukemia, while no abnormalities were detected in the hematopoietic organs of wild type animals analyzed. Npm loss of function at the centrosome has been involved in tumor development in these mice as bone marrow cytospins from blasts of affected mice clearly showed an increased number of cells displaying multiple centrosomes.

\section{Is NPMc+ an oncogene in vitro?}

Until very recently there was no published evidence that NPMc+ would transform primary cells in vitro. At first, the NPMc+ oncogenic capacity in vitro has been debated as a study suggested a tumor suppressive role for the NPMc+ mutant because its overexpression in ARF null cells end up inhibiting cell proliferation. ${ }^{8}$

In order to establish whether NPMc+ is an oncogene in vitro, the effect of NPMc+ overexpression in primary MEFs has been studied performing classical oncogenic transformation assays in soft agar. ${ }^{9}$ The study demonstrated that NPMc+ is able to transform when it is combined with E1A. E1A is an adenoviral oncogene that induces a E2F1-dependent cell cycle progression. At the same time it induces apoptosis through Arf and p53. NPMc+ blocks Arf, allowing E1A to evade the p53 induced apoptosis in transformed cells while the addition of E1A completely abolishes the NPMc+ induced senescent phenotype and allows transformation. This represented the first demonstration that NPMc+ is an oncogene in vitro and suggested the need for a cooperative event in leukemogeneis.

Data arising from other studies described a model in which mutations of NPM1 seem to simultaneously dampen a tumor-suppressor pathway $^{4}$ (p53-ARF) and enhance an oncogenic one (MYC). ${ }^{5}$ In fact, NPMc+ was demonstrated to be able to reduce the ability of ARF to initiate a p53 response and to induce cell cycle arrest. Bonetti et al. show that, in the absence of NPM or in the presence of NPMc+, FBW7 $\gamma$ loses its nucleolar localization and is rapidly degraded by the proteasome. FBW7 $\gamma$ is a nucleolar ubiquitin ligase previously implicated in the ubiquitination/degradation of MYC. $\mathrm{NPMc}+$ maintains the property of interacting with FBW7 $\gamma$ but delocalizes it to the cytoplasm, where it is degraded, thus leading to accumulation of MYC and increased MYC signaling.

The NPM1 mutant may also exert its transforming properties through gain-of function in cytoplasm. ${ }^{6}$ Interestingly, the NPM1 mutant binds caspase 6 and 8 in the cytoplasm and specifically inhibits the activities of these celldeath caspases through direct interaction with their cleaved active forms. Moreover NPMc+ is able to suppress caspase 6 and 8-mediated myeloid differentiation.

\section{Is NPMc an oncogene in vivo?}

Until very recently there was no published data on NPMc+ oncogenic activity in vivo. However, cytoplasmic mutated NPM has been retained for eight years in a xenotransplant model of NPMc+ acute myeloid leukemia in immunodeficient mice by subcutaneous injection of AML blasts from a patient. ${ }^{10}$ This study demonstrated that cytoplasmic mutated NPM1 is stable in AML and suggested that the NPM1 mutation represent a founder genetic lesion in AML.

In an attempt to understand the in vivo role of NPMc+ in AML, it has been recently developed a model showing an impact of the mutation on myelopoiesis with no progression toward overt leukemia.11 NPMc+ was overexpressed under the control of the MRP8 promoter which lead the expression of NPMc+ in the common myeloid progenitors as well as mature granulocytes and monocytes. These mice came down with myeloproliferative disorders with splenomegaly and increased number of Gr1Mac-1 mature myeloid cells in the bone marrow and spleen. However, the latency was long and the phenotype was not fully penetrant among the different line analyzed and, more importantly, the NPM1 mutant alone was not able to initiate AML. Consequently, to exert its oncogenic effect, NPM1 may need to act under different conditions, such as targeting a specific stem cell/myeloid precursor and/or achieving a mutant to wild-type expression ratio that is appropriate for cytoplasmic delocalization of both nucleophosmin forms and/or being accompanied by a secondary cooperating event. However, the fact that NPMc+ is a bona fide oncogene in vivo comes from another interesting finding that NPMc+ also drives epithelial cancer given the fact that MRP8 is a leaky promoter in skin. Considering MRP8 is leaky in the keratinocytes, this presumably demonstrates that NPMc+ is an oncogene in vivo.

Data on zebrafish completely confirm what shown in the MRP8-NPMc+ transgenic mouse model. ${ }^{12}$ In zebrafish, ubiquitous mutant $\mathrm{NPMc}+$ not only caused expansion of primitive myeloid cells, but also resulted in increased numbers of definitive erythro-myeloid progenitors (gata1+/lmo2bright) and hematopoietic stem cells $(c-m y b+/ c d 41+)$ in the aorta ventral wall. In the zebrafish embryo, follow-up for AML development was not possible due to the transient nature of mutant NPM1 expression.

More recently a conditional knock-in allele of the NPM1 mutation A demonstrated that $\mathrm{NPMc}+$ causes acute myeloid leukemia in mice. ${ }^{13} \mathrm{NPMc}+$ heterozygous activation in the hematopoietic system was associated with expansion of mature bone marrow mature myeloid cells as previously described. Knock-in mutant display increased incidence of acute myeloid and lymphoid leukemias arising with a long latency suggesting the requirement for additional mutations. Insertional mutagenesis screens in vivo accelerated leukemia onset confirming the need for cooperative events in $\mathrm{NPMc}+$ leukemogenesis.

\section{Conclusions}

The potential role of the NPM mutant as oncogene as well as the aberrant mechanism that leads to leukaemogenesis in NPMc+ AML is still under investigation. Here we have presented the most relevant studies describing how NPM1 alterations could contribute to leukemia. NPM1 expression and gene integrity are frequently altered in human hematological malignancies. Thus, the pathogenesis of NPMc+ AML has been proposed to depend on both NPM functional loss and the NPMc+ mutant oncogenic activity.

Genetic and molecular evidence has shed light on the mechanisms of NPM-mediated tumor suppression, demonstrating that Npm is a haploinsufficient suppressor of both myeloid and lymphoid malignancies in the mouse. $\mathrm{NPMc}+$ is able to exert oncogenic potential by either loss or gain of function effects that impact on multiple cellular pathways. It has been demonstrated that NPMc+ act as a bona fide proto-oncogene in vitro and in vivo and suggested that additional cooperative genetic hits are required for leukemogenesis. Knock-in mouse models mimicking human NPM1-mutated AML are still under development and will be essential tools in the discovery of novel $\mathrm{NPMc}+$ leukemogenic activities.

\section{References}

1. Grisendi S, Mecucci C, Falini B, Pandolfi PP. Nucleophosmin and cancer. Nat Rev Cancer 2006;6:493-505.

2. Falini B, Mecucci C, Tiacci E, et al. Cytoplasmic nucleophosmin in acute myelogenous leukemia with a normal karyotype. $\mathrm{N}$ Engl J Med 2005;352:254-66.

3. Grisendi S, Bernardi R, Rossi M, et al. Role of nucleophosmin in embryonic development and tumorigenesis. Nature 2005;437:147-53.

4. Colombo E, Martinelli P, Zamponi R, et al. Delocalization and destabilization of the Arf tumor suppressor by the leukemia-associated NPM mutant. Cancer Res 2006; 66:3044-50.

5. Bonetti P, Davoli T, Sironi C, et al. Nucleophosmin and its AML-associated mutant regulate c-Myc turnover through $\mathrm{Fbw} 7$ gamma. J Cell Biol 2008;182:19-26. 
6. Leong SM, Tan BX, Bte Ahmad B, et al. Mutant nucleophosmin deregulates cell death and myeloid differentiation through excessive caspase- 6 and -8 inhibition. Blood 2010;116:3286-96.

7. Sportoletti P, Grisendi S, Majid SM, et al. Npml is a haploinsufficient suppressor of myeloid and lymphoid malignancies in the mouse. Blood 2008;111:3859-62.

8. den Besten W, Kuo ML, Williams RT, Sherr CJ. Myeloid leukemia-associated nucleophosmin mutants perturb p53-dependent and independent activities of the Arf tumor suppressor protein. Cell Cycle
2005;4:1593-8.

9. Cheng K, Grisendi S, Clohessy JG, et al. The leukemia-associated cytoplasmic nucleophosmin mutant is an oncogene with paradoxical functions: Arf inactivation and induction of cellular senescence. Oncogene 2007;26:7391-400.

10. Falini B, Martelli MP, Mecucci C, et al. Cytoplasmic mutated nucleophosmin is stable in primary leukemic cells and in a xenotransplant model of NPMc+ acute myeloid leukemia in SCID mice. Haematologica 2008;93:775-9.

11. Cheng K, Sportoletti P, Ito K, et al. The cy- toplasmic NPM mutant induces myeloproliferation in a transgenic mouse model. Blood 2010;115:3341-5.

12. Bolli N, Payne EM, Grabher C, et al. Expression of the cytoplasmic NPM1 mutant (NPMc+) causes the expansion of hematopoietic cells in zebrafish. Blood 2010;115:3329-40.

13. Vassiliou G, Cooper JL, Rad R, et al. A conditional knock-in allele of the type a cytoplasmic nucleophosmin mutationcooperates with a novel sleeping beauty transposon to cause acute myeloid leukaemia in mice. Haematologica 2010; 95(s2):240, abstr. 0578. 